
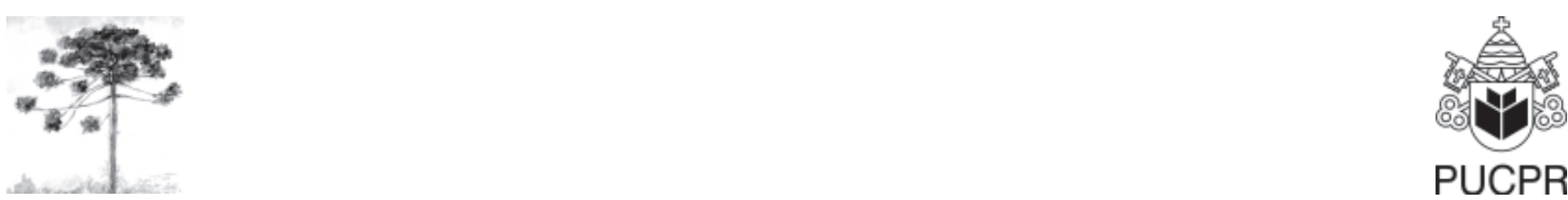

\title{
ESTRUTURA POPULACIONAL DE Bradypus tridactylus (XENARTHRA, BRADYPODIDAE) EM FRAGMENTO FLORESTAL URBANO NO MUNICÍPIO DE MANAUS, AMAZONAS, BRASIL
}

\author{
Population structure of Bradypus tridactylus (Xenarthra, Bradypodidae) \\ in Manaus forest urban fragment, Amazonas State, Brazil
}

\section{Carla Letícia Réus ${ }^{[1]}$, Cristiano Martins de Souza ${ }^{[2]}$}

${ }^{[1]}$ MSc. em Biologia Animal pela Universidade Federal do Rio Grande do Sul (UFRGS), Porto Alegre, RS - Brasil, e-mail: carlalreus@yahoo.com.br

${ }^{[2]}$ MSc. em Ecologia pelo Instituto Nacional de Pesquisas da Amazônia (INPA), Manaus, AM-Brasil, e-mail: cris.ms@click21.com.br

\section{Resumo}

Os objetivos deste trabalho foram estimar a população de Bradypus tridactylus Linnaeus, 1758 dentro de um fragmento; verificar a proporção sexual e a faixa etária (indivíduos adultos [machos e fêmeas], jovens e filhotes) e sugerir, com base nos dados coletados, se há uma superpopulação desses animais na área de estudo. O local de estudo foi o Bosque da Ciência, uma área de 10 ha pertencente ao Instituto Nacional de Pesquisas da Amazônia. O método utilizado foi o Método de Contagem Direta em um transecto de $1.500 \mathrm{~m}$ de comprimento nas trilhas do bosque, sendo verificada uma distância de $10 \mathrm{~m}$ para cada lado (bordas). Observou-se, num período de 15 dias consecutivos, um total de 38 indivíduos de B. tridactylus, sendo machos $(\mathrm{n}=11)$, fêmeas $(\mathrm{n}=21)$, jovens $(n=03)$ e filhotes $(n=01)$. A proporção sexual da população observada foi de um macho para cada 1,9 fêmeas. O resultado de 3,8 ind/ha é bastante alto quando comparado a outros resultados obtidos em fragmentos, como na Estação Ecológica do Mamirauá (2,12 ind/ha) e no fragmento florestal da Universidade Federal do Amazonas que, mesmo estando localizada bem próxima ao Bosque da Ciência, apresenta uma notória diferença em densidades de preguiças $(2,2$ ind/ha). A falta de predadores e a alta disponibilidade de alimento (principalmente Cecropia sp.) no fragmento em estudo pode ocasionar uma superpopulação de B. tridactylus.

Palavras-chave: Preguiça-bentinho; Preguiça-de-três-dedos; Edentata. 


\begin{abstract}
The objectives of this work: to esteem the population of Bradypus tridactylus Linnaeus, 1758 inside of one I break up; to verify the sexual ratio and band of age (adult individuals (male and female), young and younglings) and to determine, on the basis of the collected information, if has an overpopulation of these animals in the area of study. The study place was the Bosque da Ciência, a pertaining area of 10ha to the National Institute of Research of Amazonia. The used method was the method of direct count in a line of $1.500 \mathrm{~m}$ of length in the tracks of the forest, being verified a distance of $10 \mathrm{~m}$ for each side (edges). They have been observed, in a period of 15 consecutive days a total of 38 individuals of B. tridactylus being males $(n=11)$, females $(n=21)$, young $(n=03)$ and younglings $(n=01)$. The sexual ratio of the observed population was of one male for each 1,9 females. The result of 3,8 ind/ha is sufficiently high when compared with other results gotten in forests break ups, with in the Ecological Station of Mamiraua $(2,12 \mathrm{ind} / \mathrm{ha})$ and in I break up it forest of the Federal University of Amazon, that exactly being located nearness to the Bosque da Ciencia well, it presents a wellknown difference in sloth densities (2,2 ind/ha). The lack of predators and the high food availability (mainly Cecropia sp.) in I break up in study can cause it an overpopulation of B. tridactylus.
\end{abstract}

Keywords: Sloth; Edentata.

\section{INTRODUÇÃO}

O desenvolvimento dos mamíferos iniciouse por volta de 135 milhões de anos atrás, sendo que a radiaçãodos Eutheria teveinício somente após a radiação adaptativa dos marsupiais (Metatheria) (1). O primeiro ramo foi o dos Edentata, surgido há cerca de 50 milhões de anos (2). Nesta fase, os continentes que seriam posteriormente as Américas do Sul e do Norte já haviam se separadoda Áfricaeda Ásia, respectivamente. Este isolamento foi vital para o desenvolvimento de formas especializadas como os Edentata (3).

Do ramo dos Pillosa, que constituem as preguiças e os tamanduás, originou-se a superfamília Phyllophaga, representada pelas preguiças extintas e recentes (4). Estas agruparam-se em quatro principais famílias: Mylodontidae, Megatheriidae, Megalonychidae e Bradypodidae (5); as três primeiras continham representantes arborícolas e terrestres, pesando entre 500 e $4.000 \mathrm{~kg}$, enquanto a última, apenas arborícolas (6, 7). Entretanto, com a formação do istmo do Panamá, há cerca de 3 milhões de anos, houve inicial intercâmbio entre as duas Américas e posterior extinção de gêneros e famílias em ambas as massas continentais (8).

Atualmente, há apenas um gênero representando a família Megalonychidae (Choloepus Illiger, 1811), e outro representando a família Bradypodidae (BradypusLinnaeus, 1758) (4), embora alguns autores sugiram que esses dois gêneros devam ser colocados dentro da mesma família,
Bradypodidae (9). A diferença anatômica mais marcante entre os gêneros é a presença de três dedos ou unhas em Bradypus e apenas dois em Choloepus $(4,10)$. Estes animais ocorrem na região Neotropical, sobretudo nas florestas equatoriais e subequatoriais úmidas, ou em matas abertas e regiões próximas a rios. Bradypus e Choloepus possuem três e duas espécies, respectivamente (11).

Bradypus são mamíferos arborícolas de tamanho médio, cujos pelos do dorso não ultrapassam $50 \mathrm{~mm}$ e apresentam relação simbiótica com algas verdes, que cobrem grande parte de sua superfície. Franco (12) sugere que essas algas contribuem para que os animais se confundam com o ambiente ao seu redor. Possuem reduzido número de peças dentais, apenas nove pares de dentes, não possuem incisivos e caninos, pré-molares e molares não são diferenciados, sendo igualmente pequenos. A cauda é pequena, com aproximadamente $70 \mathrm{~mm}$ e o trato digestório é dilatado, com cerca de $30 \%$ do peso vivo do animal. A alta taxa de passagem do alimento, que permite maior aproveitamento da ingesta, e a comparativa ineficiência energética do processo fermentativo exigem uma taxa de metabolismo basal relativamente baixa $(7,13)$.

Dentro deste gênero encontra-se a preguiçabentinho (B. tridactylus), uma das mais afetadas pela fragmentação florestal por causa de seus hábitos e baixo metabolismo, que fazem com que permaneça em pequenas áreas fragmentadas até o esgotamento total dos recursos. SegundoSilva \& Rylands (14), a capacidade 
de uma determinada área abrigar espécies está diretamente relacionada à disponibilidade destes. Quando alguma espécie fica impossibilitada de migrar em busca de novos recursos e procura dirigir-se a outras áreas, estes podem não ser facilmente encontrados, comprometendo o futuro desta população $(4,7)$.

Bradypus tridactylus Linnaeus, 1758 tem distribuição geográfica nas Guianas, Suriname, Guiana Francesa, Venezuela e Norte do Brasil (região do Rio Solimões Amazonas) (3, 15).

$\mathrm{Nas}$ áreas tropicais, a taxa de desmatamento excede 15 milhões de hectares anualmente, resultando na extensiva fragmentação da floresta (16) que, aliada à falta de conhecimento dos efeitos da fragmentação sobre os organismos, torna ainda mais grave esse problema $(17,18)$.

A fragmentação é invariavelmente destrutiva para ambientes naturais e resulta em perda e alteração dos hábitats (18-20), aumento do efeito de borda $(19,21)$, aumento do isolamento de populações florestais, aumento no fluxo de indivíduos provindos de áreas destruídas e/ou alteradas, além de propiciar invasões dos fragmentos por espécies exóticas ou favorecidas por hábitats perturbados (19). A redução e fragmentação de hábitats forçam os indivíduos a ocupar uma área menor que a ocupada originalmente, aumentando a competição e a frequência de encontros agressivos, induzindo assim a mortalidade e emigração (22).

Um exemplo da fragilidade deste grupo é a preguiça de coleira, Bradypus torquatus Illiger, 1811, espécie endêmica da Floresta Atlântica do sul da Bahia, Espírito Santo e Rio de Janeiro, a qual se encontra na lista dos mamíferos brasileiros ameaçados de extinção $(23,24)$. Outros fatores tornam as preguiças ainda mais vulneráveis, como a caça e suas características reprodutivas, com longos períodos de gestação (seis meses) e nascimento de um único filhote por vez, que fica dependente da mãe por longo período $(4,15)$.

Poucos trabalhos sobre $B$. tridactylus foram realizados até hoje, e verificaraspectos como o tamanho populacional e a área mínima de vida, principalmente em fragmentos florestais, são de grande importância para a conservação desses animais. Por essa razão, o objetivo deste trabalho é estimar a densidade populacional de B. tridactylus dentro de um fragmento florestal urbano na cidade de Manaus, além de verificar a proporção sexual (adultos machos e fêmeas) e a faixa etária (indivíduos adultos, jovens e filhotes) dos indivíduos avistados; com base nos dados coletados e comparação com outros estudos, sugerir se há superpopulação desses animais na área estudada.

\section{MATERIAIS E MÉTODOS}

Opresente estudo foi realizado em fragmento florestal urbano pertencente ao Instituto Nacional de Pesquisas da Amazônia (INPA), denominado Bosque da Ciência (Figura 1). Este local foi inicialmente criado com o objetivo de divulgar a fauna e flora da região e tratar de projetos de educação ambiental.

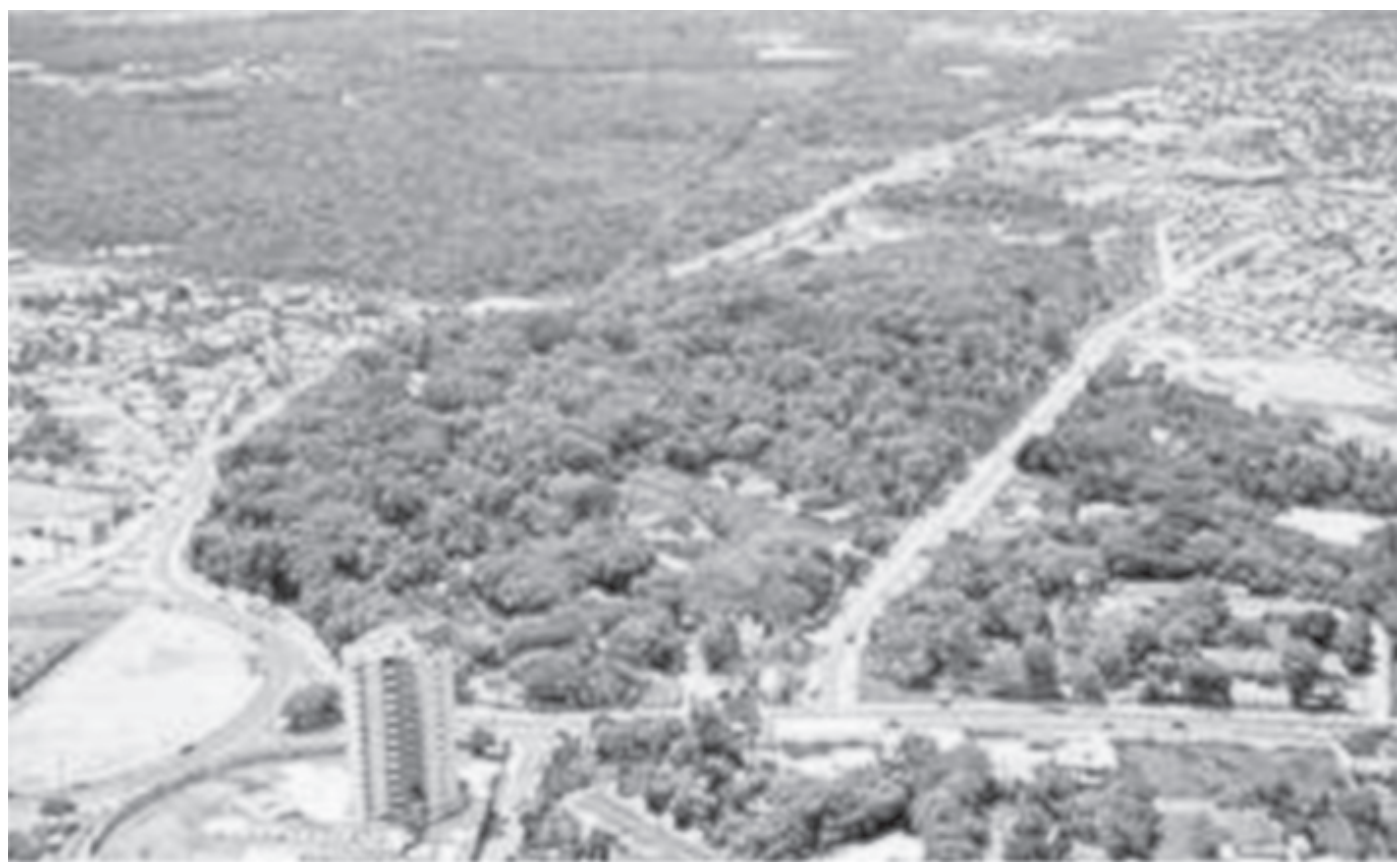

FIGURA 1 - Fotografia da área do fragmento do Bosque da Ciência - INPA (ao centro) e parte da área verde da Universidade Federal do Amazonas - UFAM (ao fundo) 
O Bosque está situado dentro da área do Instituto, na cidade de Manaus, Estado do Amazonas. O INPA possui área total de $130.000 \mathrm{~m}^{2}$, sendo que $100.000 \mathrm{~m}^{2}$ são de área verde e formam o Bosque da Ciência. O clima é tropical úmido com uma estação seca que se estende de maio a novembro e uma estação chuvosa que vai de dezembro a abril.

O Bosque da Ciência possui trilhas internas que possibilitam a cobertura de boa parte da área. As coletas de informações ocorreram por meio de censo (25), utilizando o método de contagem direta (MCD) proposto por Brower e Zar (26), que sugerem que animais sésseis ou de movimentos lentos sejam recenseados da mesma forma que os vegetais, ou seja, pelo MCD: numa área de tamanho conhecido, contam-se todos os indivíduos presentes. A contagem foi realizada em um transecto de $1.500 \mathrm{~m}$ de comprimento, sendo verificada uma distância de $10 \mathrm{~m}$ para cada lado (bordas), da seguinte forma: 1) Traçou-se um itinerário dentro do sistema de trilhas que possibilitasse a cobertura da maior área possível do bosque (Figura 2); 2) O trajeto foi percorrido durante quinze dias consecutivos para que o avistamento e a contagem dos indivíduos fossem realizados; 3) A distância ao lado das trilhas para a contagem dos indivíduos foi de 10 metros, fora da qual os avistamentos não foram considerados; 4) Estipulou-se a velocidade média de $0,14 \mathrm{~m} / \mathrm{s}(1.500 \mathrm{~m} / 3 \mathrm{~h})$ para percorrer o trajeto durante toda amostragem; 5) A trilha foi devidamente medida e marcada a cada dez metros com marcadores biodegradáveis (banding tags); 6) Dados como a posição em que o animal foi avistado e o estágio de desenvolvimento (adulto, jovem ou filhote) foram anotados.

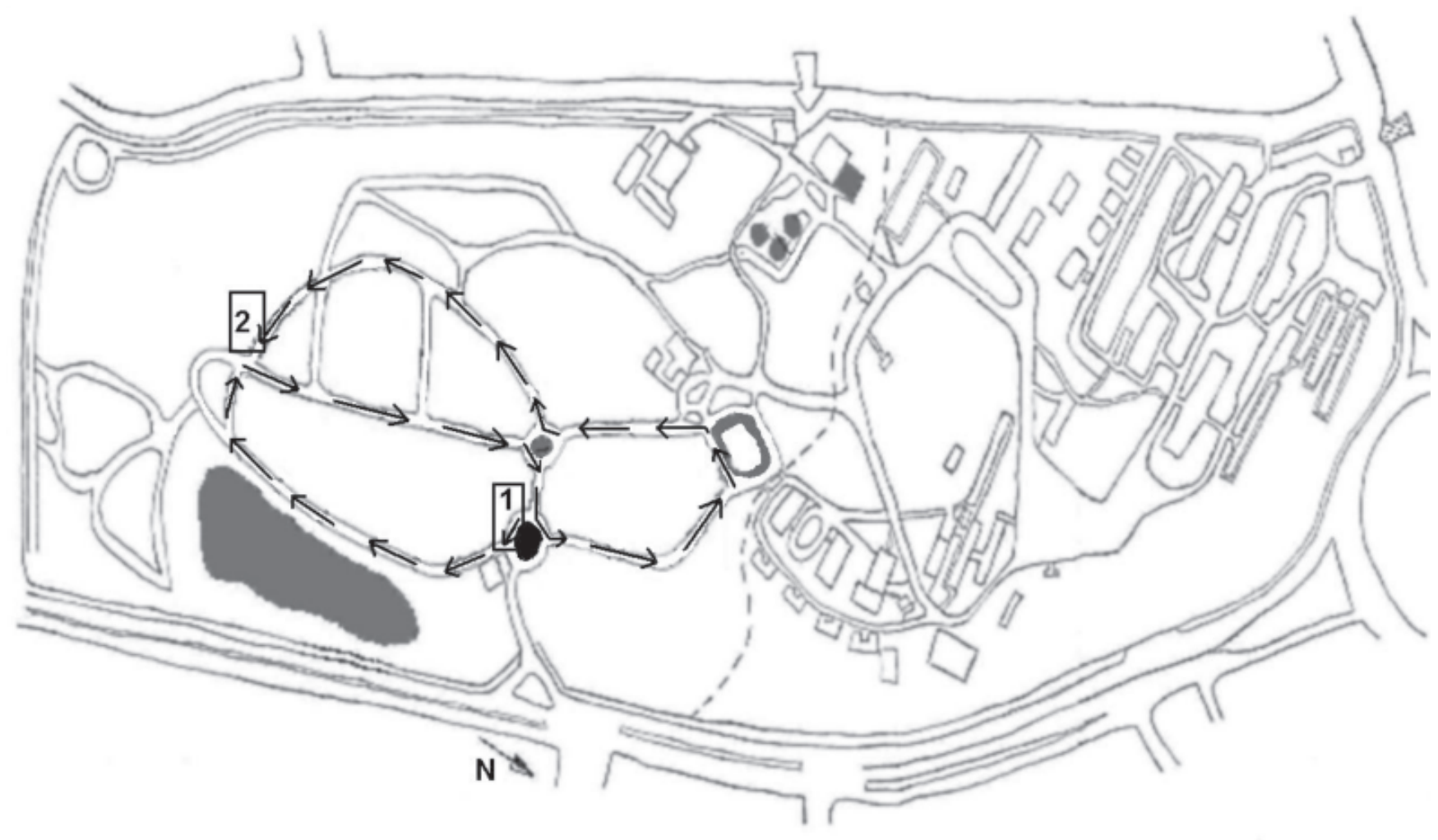

FIGURA 2 - Mapa esquemático do INPA, com itinerário percorrido destacado nas trilhas.

Nota: 1) Início da transecção; 2) Término da transecção. A linha tracejada indica divisão entre área edificada e bosque. 
Com relação aos indivíduos adultos, verificou-se o sexo do animal. De acordo com Carmo (13), apenas adultos possuem diferenciação sexual visível, já que os machos apresentam uma mancha dorsal amarelo-laranja.

O recenseamento de preguiças não é uma atividade tão usual como o de primatas. Em estudo sobre a densidade e diversidade da mastofauna em sete diferentes localidades da Amazônia, Emmons (27) só apresenta dados sobre preguiças em três dessas localidades, mostrando como o Método do Transecto Linear não é apropriado para esses animais. Os problemas encontrados pela autora foram a alta cripticidade das preguiças e a ausência de vocalização típica que denuncie a presença dos animais. Para contornar esse tipo de problema, Eisenberg e Thorington (28) propuseram a utilização do Método de Contagem de Sinais ou Vestígios (MCSV), sugerindo a contagem de montes de fezes numa área com superfície previamente conhecida, porém, os resultados obtidos com este método são frequentemente questionados, pois a área amostral geralmente é muito pequena, além disso, esse método não poderia ser aplicado à várzea, com o seu característico regime anual de alagamento.

\section{RESULTADOS E DISCUSSÃO}

Bradypus tridactylus é uma espécie pouco estudada, mesmo sendo encontrada com muita frequência na região de Manaus, especialmente nos remanescentes florestais dentro da cidade e em áreas rurais. Observou-se 38 indivíduos de B. tridactylus, sendo 11 machos, 21 fêmeas, três jovens e apenas um filhote.

Da área total do bosque $\left(100.000 \mathrm{~m}^{2}\right.$ ou $10 \mathrm{ha})$ cobriu-se $3 \mathrm{ha}\left(30.000 \mathrm{~m}^{2}=1500 \mathrm{~m} \times 20 \mathrm{~m}\right)$ por dia de observação, sendo encontrada uma densidade média de 12,66 ind/ha (indivíduos por hectare). Esse valor é alto quando comparado a outros resultados obtidos em fragmentos, como na Estação Ecológica do Mamirauá (EEM) (2,12 ind/ha) (4) e no fragmento florestal da Universidade Federal do Amazonas (UFAM), que mesmo estando localizada bem próxima ao Bosque da Ciência, apresenta diferença significativa na densidade de preguiças (2,2 ind/ha) (13).

A proporção sexual da população observada foi de um macho para cada 1,9 fêmeas, assemelhando-se a Carmo (13), que no fragmento florestal da UFAM, encontrou um macho para cada 1,29 fêmeas (Tabela 1).

TABELA 1 - Dados comparativos entre estudos com o gênero Bradypus

\begin{tabular}{llll}
\hline & EEM (1995) & UFAM (2002) & INPA (2004) \\
\hline TOTAL & 22 & 53 & 38 \\
MACHOS & - & 18 & 11 \\
FÊMEAS & - & 25 & 21 \\
JOVENS & - & 02 & 03 \\
FILHOTES & - & 08 & 01 \\
TEMPO DE PESQUISA & 12 meses & 04 meses & 15 dias \\
ÁREA & 11,8 ha & 24 ha & 03 ha \\
ESPÉCIE & B. variegatus & B. tridactylus & B. tridactylus \\
MÉTODO & Transecto linear/ & Gride (12 de 2 ha cada)/ & Transecto $1.500 \mathrm{~m}$ x 20m/ \\
& Método de Contagem & Método de Contagem & Método de Contagem \\
RESULTADO & Direta (MCD) & Direta (MCD) & Direta (MCD) \\
& 2,12 ind/ha & 2,2 ind/ha & 12,66 ind/ha \\
\hline
\end{tabular}


O número de indivíduos encontrados de um dia para o outro variou entre zero e oito. Sabese que os animais da família Bradypodidae possuem hábito solitário, cuja interação social se restringe à associação mãe-filhote, que ocorre durante os primeiros meses de vida, ou ao encontro de machos e fêmeas no período reprodutivo $(4,29,30)$. Eisenberg (31) prefere nomear esta estrutura como "social não coesiva". Conforme esperado, todos os indivíduos, com exceção da mãe com o filhote, foram encontrados isolados, o que pode indicar que os animais não estavam em período reprodutivo. Cabe salientar que não existem dados na literatura sobre atividade reprodutiva de $B$. tridactylus.

Dos 38 indivíduos avistados, 18 encontravam-se em movimento, dentre estes, nove estavam em deslocamento, sendo encontrados no dia seguinte de observação, em local próximo ao do dia anterior. Este dado confirma que B. tridactylus é ativa tanto à noite quanto durante o dia, concentrando seus deslocamentos no período noturno $(7,13)$. As atividades e posições dos indivíduos avistados foram: andar, subir em árvores ou cipós, alimentar-se, pendurar-se, em repouso "embolado" ou com o corpo esticado, coçar-se e migrar para outra fonte de alimento.

De acordo com Queiroz (4), o caráter social de um grupo de primatas lhes confere adiantado grau de sincronia na execução dos diferentes comportamentos ao longo do seu período de atividades, as preguiças, ao contrário, estando separadas de seus coespecíficos, podem ou não realizar tal sincronia. Sunquist e Montgomery (32) mostraram que não havia nenhuma sincronização entre o ciclo de atividade e repouso de uma população de preguiças; contudo, não puderam afirmar se aqueles animais que se encontravam ativos num mesmo corte do tempo estavam ou não executando a mesma atividade porque tais animais não estão coesos numa unidade social ou num aglomerado, são extremamente crípticos e silenciosos e ainda não possuíam observações diretas na natureza.

Não houve relação entre o número de indivíduos e o tempo (nublado, ensolarado, chuvoso, com ou sem vento...), como também não houve nenhuma morte comprovada na população estudada de B. tridactylus durante o período de observação.

Com frequência, animais são capturados pela população e soltos em áreas verdes de parques. Essas transferências ocorrem sem nenhum embasamento científico sobre a espécie ou status das populações locais, que poderiam indicar o melhor procedimento a ser adotado em relação a este "excedente". Portanto, informações ecológicas e comportamentais como a área de vida, deslocamentos, alimentação, reprodução, entre outros, são imprescindíveis para o manejo adequado e conservação da espécie na região de Manaus (13).

As únicas informações disponíveis sobre predação de preguiças são pelotas com pelos desses animais encontrados em ninhos de Harpya harpya (gavião-real), e indícios de possível evento de predação por sucuri (Eunectes sp.) na Estação Ecológica do Mamirauá (4).

\section{CONCLUSÃO}

Houve maior número de registros de fêmeas. Todos os animais foram encontrados isolados de outros, com exceção da mãe com o filhote; este dado pode refletir que a espécie esteja fora do seu período reprodutivo. As atividades e posições dos indivíduos avistados foram variadas, havendo predominância de animais em movimento. Houve deslocamento de alguns indivíduos tanto à noite como durante o dia.

A falta de predadores e a alta disponibilidade de alimento, principalmente Cecropia sp., ao longo do ano no fragmento em estudo podem resultar numa superpopulação de B. tridactylus.

\section{AGRADECIMENTOS}

Agradecemos à Anita Macedo de Campos pelas sugestões, a vários revisores anônimos, à Isabel Monguilhott e ao INPA.

\section{REFERÊNCIAS}

1. Paula-Couto C. Tratado de paleomastozoologia. Rio de Janeiro: Academia Brasileira de Ciências; 1979.

2. Glass BP. History of classification and nomenclature in Xenarthra (edentata). In: Montgomery GG. editor. The evolution and ecology of armadillos, sloths and vermilinguas. Washington: Smithsonian Institution Press; 1985. p. 1-3. 
3. Eisenberg JF, Rerdford KH. Mammals of the Neotropics. The Central Neotropics 3. Ecuador, Peru, Bolivia, Brazil. Chicago: University of Chicago Press; 1999. 609 p.

4. Queiroz HL. Preguiças e guaribas: os mamíferos folívoros arborícolas do Mamirauá. Brasília: Sociedade Civil Mamirauá; 1995.

5. Engelman GF. The Phylogeny of the Xenarthra. In: Montgomery GG. editor. The evolution and ecology of armadillos, sloths and vermilinguas. Washington: Smithsonian Institution Press; 1985. p. 51-63.

6. McNab BK. Energetics, population biology, and distribution of Xenarthrans, living and extinct. In: Montgomery GG. editor. The evolution and ecology of armadillos, sloths and vermilinguas. Washington: Smithsonian Institution Press; 1985.

7. Feldhamer GA, Drickamer LC, Vessey SH, Merrit JF. Mammalogy: adaptation, diversity, ecology. 2nd ed. New York: Mc Graw Hill; 2004. $550 \mathrm{p}$.

8. Marshall LG, Raup DM, Sepkoski JJ, Webb SD. Mammalian evolution and the great american interchange. Science. 1982; 215(4538):1351-7.

9. Nowak RM. Walker's mammals of the world. 5th ed. Baltimore: The Johns Hopkins University Press; 1991.

10. Wilson DE, Reeder DM. Mammal species of the world: a taxonomic and geographic reference. Washington: Smithsonian Institution Press; 1993. 1206 p.

11. Chiarello AG. Activity budgets and ranging patterns of the Atlantic Forest maned sloth Bradypus torquatus (Xenarthra: Bradypodidae). J. Zoo (Lond). 1998;246(1):110.

12. Franco AMR. Caracterização biológica e molecular de parasitas do gênero Endotrypanum (Kinetoplastida: Trypanosomatidae). [tese]. Rio de Janeiro: Instituto Oswaldo Cruz em Biologia Celular e Molecular; 1995.
13. Carmo NAS. Distribuição, densidade e padrão de atividades de $B$. tridactylus (Mammalia, Xenarthra) em fragmento florestal na Amazônia Central. [dissertação]. Manaus: Universidade Federal do Amazonas; 2002.

14. Silva MNF, Rylands JL. Biogeografia e conservação da mastofauna na floresta amazônica brasileira. In: Capobianco JPR, Veríssimo A, Moreira D, Sawner I, Santos LP. editores. Biodiversidade na amazônia brasileira. São Paulo: Estação liberdade, Inst. Sócioambiental; 2001. p. 110-31.

15. Emmons LH, Feer F. Neotropical rainforest mammals: a field guide. Chicago: The University of Chicago Press; 1997. 392 p.

16. Whitmore L. Tropical forest disturbance, disappearance, and species loss. In: Laurance WF, Biorregard Jr. RO. editores. Tropical forest remnants: ecology, management, and conservation of fragmented communities. Chicago: University of Chigago Press; 1997.

17. Laurance WF, Ferreira LV, Merona JMR, Laurance SG. Rain forest fragmentation and the dynamics of amazoniam tree communities. Ecology. 1998;79(6):2032-40.

18. Schweiger EW, Diffendorfer JE, Holt RD, Pierotti R, Egaines MS. The interaction of habitat fragmentation, plant, and small mammal sucession in a old field. Ecological Monographs. 2000;70(3):383-400.

19. Laurance WF, Biorregard Jr. RO, Gascon C, Didham RK, Smith AP, Lynam AJ. et al. Tropical forest fragmentation: synthesis of a diverse and dynamics discipline. In: Laurance WF, Biorregard Jr. RO. editores. Tropical forest remnants: ecology, management, and conservation of fragmented communities. Chicago: University of Chigago Press; 1997.

20. Metzger JP. Estrutura da paisagem e fragmentação: análise bibliográfica. Anais da Academia Brasileira de Ciências. 1999;71(3): 445-63.

21. Laurance WF, Yelsen E. Predicting the impacts of edge effects in fragmented habitats. Biological Conservation. 1991;55:77-92. 
22. Johannesen E, Ims RA. Modelling survival rates: habitat fragmentation and destruction in root vole experimental populations. Ecology. 1996;77(4):1196-209.

23. Rylands JL. Lista anotada dos mamíferos do Brasil. Conservation International \& Fundação Biodiversitas. Occasional Papers in Conservation Biology. 1996;4:1-38.

24. Fundação Biodiversitas. Lista das espécies ameaçadas da fauna Brasileira [Online] 2003. [citado 15 jun. 2007]. Disponível em: URL: http:/ /www.biodiversitas.org.br/f_ameaca/ consulta.asp

25. Voss RS, Emmons LH. Mammalian diversity in neotropical lowland rainforests: a preliminary assessment. Bull Amer Mus Nat Hist. 1996;230:1-115.

26. Brower JE, Zar JH. Field and laboratory methods for general ecology. 2nd ed. Iowa: Wm C. Brown Publishers; 1984.

27. Emmons LH. Geographic variation in densities and diversities of nonflying mammals in Amazonia. Biotropica.1984; 16(3):210-22.

28. Eisenberg JF, Thorington Jr. RW. A preliminary analisys of a neotropical mammal fauna. Biotropica. 1973;5(3):150-61.
29. Chiarello AG, Chivers DJ, Bassi C, Maciel MAF, Moreira LS, Bazzalo M. A translocation experiment for the conservation of maned sloths, Bradypus torquatus (Xenarthra, Bradypodidae). Biological Conservation. 2004;114(4):421-30.

30. Lara-Ruiz P, Chiarello AG. Life history traits and sexual dimorphism of the Atlantic Forest maned sloth Bradypus torquatus (Xenarthra: Bradypodidae). J Zool., Lond. 2005;267:63-73.

31. Eisenberg JF. Reproduction in two species of spider monkeys, Ateles fusciceps and $A$. geoffroyi. J Mammal. 1973;54:955-57.

32. Sunquist ME, Montgomery GG. Activity patterns and rates of movement of two- toed three- toed sloths (Choloepus hoffmani and Bradypus infuscatus). Journal of Mammalogy. 1973;54(4):946-54.

Recebido: 12/07/2007 Received: 07/12/2007

Aprovado: 20/01/2008 Approved: 01/20/2008 